Autrefois, c'était aux médecins-chefs en médecine interne et en chirurgie de se charger des patients dans les urgences des hôpitaux. Un changement essentiel a eu un impact substantiel sur la médecine d'urgence en Suisse lorsqu'un service d'urgences en partie autonome a été introduit respectivement aux HUG et à I'Hôpital cantonal saint-gallois. En 1998, I'Hôpital de l'Île s'est doté du premier service d'urgences totalement indépendant, structuré comme les autres cliniques avec un médecin-chef, des médecins ralliés au service et du personnel soignant, ainsi qu'un budget propre. Le présent article du Forum du sauvetage de la FMH décrit la mutation en marche dans la médecine d'urgence.

Dr Ernst Gähler, vice-président de la FMH, responsable du département Professions paramédicales

\title{
La médecine d'urgence hospitalière en mutation
}

\begin{abstract}
Heinz Zimmermanna, Denis Bachmann ${ }^{b}$, Pascal Berger ${ }^{c}$, Konrad Diem ${ }^{\mathrm{d}}$, Bruno Durrer ${ }^{\mathrm{e}}$, Wolfgang Ummenhofer
a Prof. Dr, Expert Consultant univ., centre d'urgences de l'Hôpital de l'lle, Berne; b Dr, directeur de clinique adjoint, clinique médicale pédiatrique universitaire, Hôpital de I'lle, Berne; c Dr, chef de clinique, cliniques psychiatriques universitaires, centre de santé Kornhausgasse, Bâle; d Dr, spécialiste en médecine interne générale, médecin d’urgence SSMUS, cabinet médical, Ettingen; e Dr, spécialiste en médecine générale FMH, médecin d’urgence SSMUS, médecine du sport SSMS, Lauterbrunnen; f Prof. Dr, département Anesthésie, Hôpital universitaire de Bâle
\end{abstract}

Le Forum du sauvetage de la FMH a récemment publié des articles sur le thème de la médecine d'urgence préhospitalière et du sauvetage. Dans ces colonnes, nous vous éclairons sur différents aspects des centres et des services d'urgences.

Les centres d'urgences (Emergency Departments) existent depuis pratiquement 50 ans dans les pays anglosaxons. Alors qu'ils correspondent à une unité académique des cliniques universitaires, une formation spécialisée en médecine d'urgence a également été mise en place. Bien que la Suisse suive fondamentalement de très près les tendances en médecine de ces pays, une évolution semblable de la médecine d'urgence hospitalière n'a pas encore eu lieu chez nous.

\section{Autrefois, les médecins de famille étaient le premier point de contact en cas d'urgence}

Par le passé, les patients des régions urbaines ou rurales s'adressaient en premier lieu à leur médecin de famille en cas d'urgence. Après une première auscultation, celui-ci décidait si le patient serait traité chez lui ou s'il devait être adressé aux urgences d'un hôpital. Le cas échéant, les patients hospitalisés étaient en partie directement installés dans le service de la discipline concernée et non dans un service d'urgences. Dans les hôpitaux, c'était aux médecins-chefs en médecine interne ou en chirurgie de traiter ces patients, les ressources en personnel d'urgence provenant des unités concernées. Lorsqu'en 1998 [1], un état des lieux a été dressé en Suisse, des différences importantes sont apparues dans l'organisation et la qualité des services d'urgences: il n'était pas rare que de jeunes médecins-assistants inexpérimentés devaient assurer un service de 24 heures avec seulement une supervision insuffisante par des médecins spécialistes qui se sen- taient avant tout responsables de leur unité dans leur service respectif.

\section{La médecine d'urgence dans un service autonome}

L'introduction d'un service d'urgences en partie autonome aux HUG et à l'Hôpital cantonal saint-gallois a marqué un changement important pour la médecine d'urgence en Suisse, suivi par la création en 1998 du premier service des urgences autonome à l'Hôpital de l'Ile, structuré comme les autres services avec un médecin-chef, des médecins ralliés au service et du personnel soignant, ainsi qu'un budget propre. Même si ces premières étapes ont tout d'abord suscité des débats controversés, elles ont rétrospectivement posé les jalons de la médecine d'urgence hospitalière. Ces dernières années, de plus en plus de services ou de centres d'urgences avec un médecin adjoint ou un médecin-chef ont vu le jour dans des hôpitaux plus ou moins grands. Aujourd'hui, le nombre de patients qui se rendent directement aux urgences et s'adressent plus rarement à leur médecin de famille en cas d'urgence augmente, et ce principalement dans les régions urbaines. C'est une tendance que ni les milieux politiques ni le corps médical ne saluent mais n'oublions pas que de nombreux patients n'ont plus de médecin de famille et que la pénurie de médecins de famille devient réelle, si bien que les généralistes installés n'auraient de toute façon pas les disponibilités requises pour traiter tous les patients urgents.

\section{L'organisation du service d'urgences et les processus sont décisifs}

En 2011, 138 services d'urgences ont enregistré 1,619 million de visites de patients (4400 consultations/jour) [2], 
1 Osterwalder JJ. Emergency medicine in Switzerland. Ann Emerg

Med.1998;32:243-7.

2 Yersin B. Consultations dans un service d'urgence en Suisse. Obsan Bulletin 3/2013.

3 Bürgi U, Zürcher M, Sieber R, Bingisser R, Can Ü, Adam-Scott F, et al. Empfehlungen zu den Minimalvoraussetzungen einer Notfallstation.

Bull Méd suisses. 2014;95(35):1264-6 (en allemand).

Correspondance:

Prof. Dr Wolfgang

Ummenhofer

Département Anesthésie

Hôpital universitaire

CH-4031 Bâle

wolfgang.ummenhofer[at]

usb.ch soit une hausse de $26 \%$ par rapport à 2007 . Un traitement d'urgence cohérent en termes de qualité n'était guère possible dans ces services et ne l'est guère aujourd'hui encore en raison de la surcharge de travail, d'une expérience limitée et d'un manque de formation des médecins en médecine d'urgence, en particulier lors de situations critiques (temps). Le diagnostic initial, posé de manière rapide et compétente, et l'exécution des premières mesures lorsque le pronostic vital est engagé, sont devenus de plus en plus importants ces dernières années. Le concept de l'«heure d'or», qui revêt une immense importance pour le traitement des traumatismes (Advanced Trauma Life Support, ATLS), s'applique également aux patients victimes d'un accident cardiaque, d'un accident vasculaire cérébral, d'un anévrisme aortique, d'une hémorragie méningée mais aussi dans d'autres situations avec un facteur temps critique. Les urgentistes doivent définir et améliorer les processus de concert avec les spécialistes concernés. L'expérience a montré que non seulement le savoir des spécialistes revêt de l'importance mais aussi que les processus et l'organisation des services d'urgences sont décisifs. Les urgentistes ont donc ici un rôle important à jouer. La Société Suisse de Médecine d'Urgence et de Sauvetage (SSMUS) a récemment publié des recommandations concernant les exigences minimales en vue d'améliorer la qualité de ces services [3].

\section{Pour et contre un titre de spécialiste propre}

La médecine d'urgence hospitalière a sa place au sein de la SSMUS depuis 2005. En plus de l'attestation de formation complémentaire «médecin d'urgence», la FMH propose aussi depuis 2009 une AFC en médecine d'urgence hospitalière. En revanche, plusieurs sociétés de disci-

\section{Sur le fil}

A l'inverse des systèmes de santé anglo-saxons, l'évolution de la médecine d'urgence hospitalière a commencé avec beaucoup de retard en Suisse.

Principalement dans les régions urbaines, les services d'urgences remplacent aujourd'hui de plus en plus les consultations chez un médecin de famille. Cette tendance est à la hausse.

Dans les hôpitaux plus grands, il est décisif d'avoir, en plus des spécialistes, des urgentistes compétents avec des processus clairement structurés en vue de garantir une prise en charge efficace des patients urgents, notamment lors d'affections ou de blessures présentant un facteur temps critique.

La FMH propose depuis 2009 une attestation de formation complémentaire en médecine d'urgence hospitalière. La nécessité de créer un titre de spécialiste en médecine d'urgence fait actuellement débat.

Un diplôme spécialisé en soins d'urgence existe déjà pour le personnel soignant; créé sur la base des possibilités de synergies avec des formations apparentées en anesthésie et en médecine intensive.

La reconnaissance de la médecine d'urgence au niveau académique et la participation scientifique qui en découle ont augmenté également en Suisse. Une évolution que les universités honorent de plus en plus, avec notamment la formation en médecine d'urgence et des compétences en la matière pour les médecins de premier recours de demain. pline sont plutôt sceptiques quant à la création d'un titre de spécialiste en médecine d'urgence. Leur crainte de perdre une partie de leur discipline (les patients des urgences) et de ne plus pouvoir intervenir directement dans les processus des traitements urgents est compréhensible. Il appartient à la médecine d'urgence d'en tenir compte. De vifs débats et pas mal de temps seront encore nécessaires pour trouver la meilleure voie vers une prise en charge d'urgence dans l'intérêt de nos patients.

Avant nous médecins, le personnel soignant avait déjà défini une formation spécialisée et créé un profil autonome de "soins d'urgence» qui, entre temps, a été relié et en partie modulé avec deux autres cursus de formation post-diplôme apparentés en anesthésie et en médecine intensive. Des modules à thème spécifique ont également vu le jour pour les services d'urgences pédiatriques. Si la médecine d'urgence trouve très lentement sa place dans le domaine universitaire, les universités de Lausanne et de Berne ont été à l'avant-garde en créant un poste de professeur en médecine d'urgence (ordinaire pour la première et extraordinaire pour la seconde). Une recherche sur PubMed indique cependant que les publications dans le domaine de la médecine d'urgence en Suisse sont en nette augmentation.

\section{Quel avenir pour la médecine d'urgence?}

Malgré des efforts ciblés, le nombre de médecins de famille continuera de diminuer fortement ces prochaines années, obligeant par conséquent les patients à s'adresser encore plus souvent à un service ou à un centre d'urgences. Dans les régions très rurales et alpines sans hôpitaux, ce seront les généralistes et les centres de médecins de famille qui continueront de garantir demain une grande partie des soins d'urgence. De plus, le vieillissement de la population entraînera une forte augmentation des patients âgés dans le domaine de la médecine d'urgence. Davantage de médecins spécialisés seront nécessaires pour les traiter le mieux possible et ils devront être disposés à travailler souvent le soir ou le week-end. En clair, la formation et la formation médicale postgraduée en médecine d'urgence restera un sujet crucial aussi bien pour le personnel soignant que pour les médecins. La formation universitaire doit également attacher davantage d'importance à la médecine d'urgence: un projet-pilote de l'Université de Bâle visant à intégrer de manière longitudinale le catalogue des objectifs d'apprentissage du cours de médecin de garde dans les études de médecine grâce à un cursus particulier pourrait jouer un rôle précurseur. 